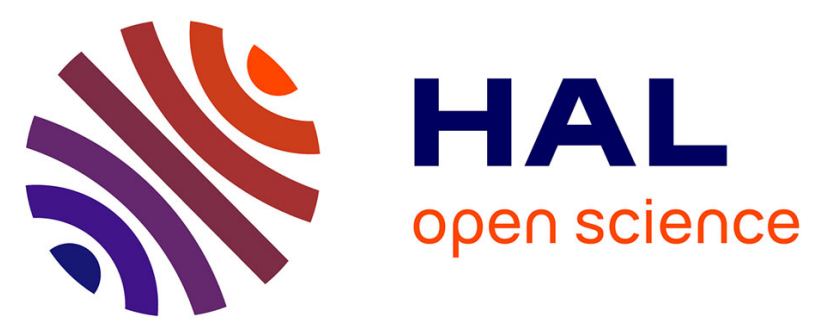

\title{
Tracking fluids in multiple scattering and highly porous materials: toward applications in non-destructive testing and seismic monitoring
}

Romain Théry, Antoine Guillemot, Odile Abraham, Éric Larose

\section{To cite this version:}

Romain Théry, Antoine Guillemot, Odile Abraham, Éric Larose. Tracking fluids in multiple scattering and highly porous materials: toward applications in non-destructive testing and seismic monitoring. Ultrasonics, 2020, 102, pp.106019. 10.1016/j.ultras.2019.106019 . hal-02376463

\section{HAL Id: hal-02376463 \\ https://hal.science/hal-02376463}

Submitted on 22 Nov 2019

HAL is a multi-disciplinary open access archive for the deposit and dissemination of scientific research documents, whether they are published or not. The documents may come from teaching and research institutions in France or abroad, or from public or private research centers.
L'archive ouverte pluridisciplinaire HAL, est destinée au dépôt et à la diffusion de documents scientifiques de niveau recherche, publiés ou non, émanant des établissements d'enseignement et de recherche français ou étrangers, des laboratoires publics ou privés. 


\title{
Tracking fluids in multiple scattering and highly porous
}

\section{materials: toward applications in non-destructive testing and}

\author{
seismic monitoring
}

\author{
Romain Théry*1, Antoine Guillemot ${ }^{2}$, Odile Abraham ${ }^{1}$, and Éric Larose ${ }^{2}$ \\ ${ }^{1}$ IFSTTAR-GERS, Laboratoire Géophysique et Évaluation Non Destructive, Bouguenais, France \\ ${ }^{2}$ Univ. Grenoble Alpes, CNRS - ISTerre - Grenoble, France
}

\begin{abstract}
Seismic and ultrasonic waves are sometimes used to track fluid injections, propagation, infiltrations in complex material, including geological and civil engineered ones. In most cases, one use the acoustic velocity changes as a proxy for water content evolution. Here we propose to test an alternative seismic or acoustic observable : the waveform decorrelation. We use a sample of compacted millimetric sand as a model medium of highly porous multiple scattering materials. We fill iteratively the sample with water, and track changes in ultrasonic waveforms acquired for each water level. We take advantage of the high sensitivity of diffuse coda waves (late arrivals) to track small water elevation in the material. We demonstrate that in the mesoscopic regime where the wavelength, the grain size and the porosity are in the same order of magnitude, Coda Wave Decorrelation (waveform change) is more sensitive to fluid injection than Coda Wave Interferometry (apparent velocity change). This observation is crucial to interpret fluid infiltration in concrete with ultrasonic record changes, as well as fluid injection in volcanoes or snow melt infiltration in rocky glaciers. In these applications, Coda Wave Decorrelation might be an extremely interesting tool for damage assessment and alert systems.
\end{abstract}

Keywords: porous media, multiple scattering, coda, fluid migration

\section{Introduction}

Tracking fluids in porous media is a common problematic to several fields of research, from geology (hydro-geology, oil prospection, $\mathrm{CO}_{2}$ sequestration, volcanology) to civil engineering (struc-

*romain.thery@ifsttar.fr 
tural health monitoring and non destructive evaluation). The goals of these researches are usually to detect, locate and quantify fluid flows. In practice, electrical and electromagnetic techniques have been largely used [Archie, 1942, Benson, 1991, Barker \& Moore, 1998, Villain et al., 2012]. Remote and light, these techniques yet encounter limits, for instance in presence of conductive materials (such as clay or metals). The many success of elastic waves in imagery, along with their relative simplicity of implementation, suggest mechanical waves as a relevant alternative. Elastic waves are indeed very sensitive to the mechanical properties of their propagation medium. Numerous papers have been published, investigating water content effects on elastic waves propagation, in various porous medium. Among them, field studies on sedimentary soils [Konstantaki et al., 2013], laboratory experiments on rocks [Winkler \& Nur, 1979] or concrete [Abraham et al., 2012, Garnier et al., 2013], which all show variations of elastic waves velocities and attenuation with water content. These variations aren't always monotonic, and differ according to the type of media. However, these studies consider ballistic wave propagation. Indeed, wavelength are significantly larger than pore or grain size.

In this paper we use multiply scattered waves, with wavelength smaller or equal to the pore size, in order to detect changes induced by fluid infiltrating in porous media. These waves, also named coda-waves, spend a larger amount of time propagating through the medium, and hence interact more with changes. This high sensitivity of coda waves has been proven [Snieder et al., 2002] and used in many applications, from volcanoes and fault zones monitoring [Sens-Schönfelder \& Wegler, 2006, Brenguier et al., 2008] to crack detection in concrete [Zhang et al., 2012, Zhang et al., 2016, Zhang et al., 2018]. Coda Wave interferometry (CWI) is a coda-based technique permitting to extract an apparent velocity change in the media, whereas Coda Wave Decorrelation (CWD) allows to assess and quantify structural or geometrical changes. [Grêt et al., 2006] already showed that CWI is sensitive to fluid presence in sandstone. We here present the results of an experiment aiming to track an increasing water front in concrete, using CWI and CWD. We then use these results to interpret those from a similar experiment in concrete, along with observations made on volcanoes and rocky glacier.

\section{Monitoring of water level elevation in sand}

We here present a laboratory experiment aiming at seeing the effects of a water front, ascending in a compacted and pre-humidified sample of sand, on ultrasonic coda waves emitted and recorded beneath its surface. We show in Fig. 1 the experimental setup. Millimetric bioclastic limestone sand is humidified, placed in a cylindrical $(13 \mathrm{~cm}$ in diameter) glass container, and 
compacted. The final height of the sample is about $8 \mathrm{~cm}$. The pre-humidification part allows us to only consider water saturation - or water content - variations as the causes of change in wave propagation. It is indeed known that for weak water saturation values, the impact of water potential - or capillary effects - on wave propagation is as important as the one of water content [Lu \& Sabatier, 2009]. A simple set-up makes it possible to introduce water in the bottom of the container. Water is iteratively added and its height is visually controlled through the glass wall.

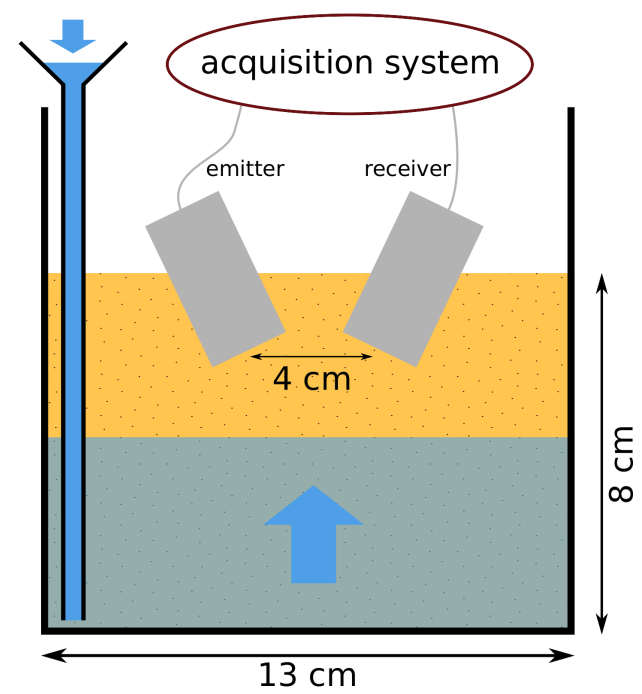

Figure 1: Set-up of the experiment.

A water front ascending in a pre-humidified and compacted sand sample is monitored using ultrasonic coda.

Two ultrasonic transducers used in the emitter-receiver configuration, are buried $2 \mathrm{~cm}$ below the surface of the sample. Impulse responses are retrieved for each incremental water level step, by correlating the measured signal (averaged over 200 acquisitions) with the source signal (linear chirp from $200 \mathrm{kHz}$ to $400 \mathrm{kHz}$ ).

Acquired impulse responses (Fig. 2) show no distinct first arrival. Indeed in our experiment, the grain size $S$ is ranging from $0.1 \mathrm{~mm}$ to $2 \mathrm{~mm}$, and the wavelength $\lambda$ is of the order of a few millimeters. According to litterature [Jia, 2004, Jia et al., 2009] we can estimate the scattering mean free path $l^{\star}$ to be ranging from approximately a few millimeters to a few centimeters. Furthermore, the size of the medium $H$ is $12 \mathrm{~cm}$ (twice the distance separating the sensors from the bottom of the container). Thus we are in the multiple scattering regime, where $S<\lambda<l^{\star}<H$.

Although coda's complex waveform could be interpreted as noise, it is in fact a deterministic and reproducible signal. Furthermore, coda waves spend more time than ballistic ones in the medium, hence interacting more often with possible developing changes. This high sensitivity 


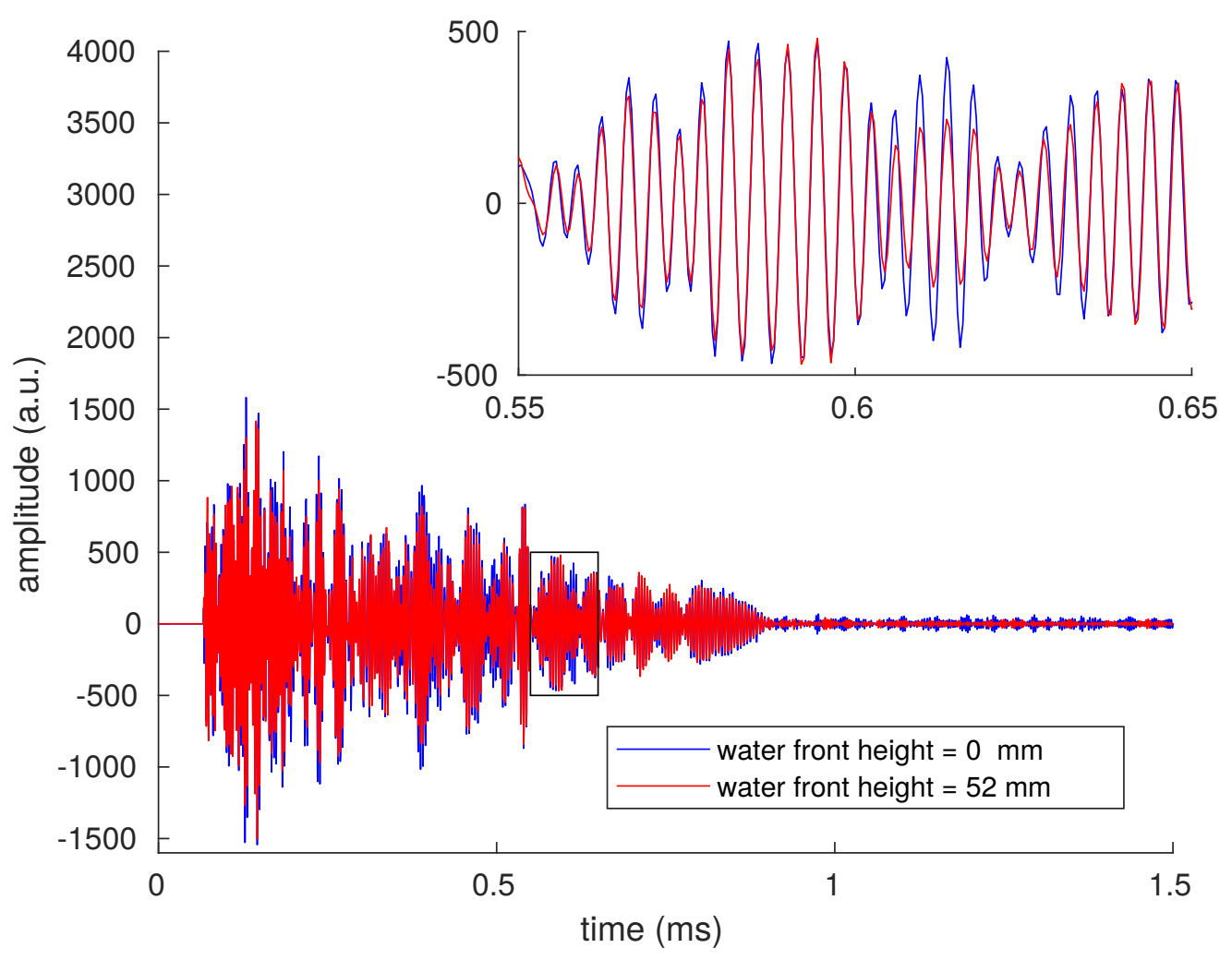

Figure 2: Impulse responses measured before and during imbibition. First amplitude values of the signals have been manually set to zero, in order to mask a single peak corresponding to cross-talk in the recordings. This modification only aims to simplify the figure without any consequences on our results, since early arrivals aren't studied here. Inset shows a close-up around the center of the observation time window used in the following. Although waveforms are different, phase shifts are hardly visible.

We then compare each impulse response with the reference one (recorded before addition of water). Hence using the reproducibility property of coda waves, we apply the stretching technique [Lobkis \& Weaver, 2003, Sens-Schönfelder \& Wegler, 2006, Larose \& Hall, 2009] to track phase and waveform changes in the signal, with increasing water level. Let us consider the reference signal, called $\varphi$, and the perturbed one, called $\varphi^{\prime}$. We stretch the reference signal from a factor $\epsilon$ and then compute the correlation coefficient between the stretched reference signal and the perturbed one (Eq. 1) :

$$
C C(\epsilon)=\frac{\int_{t_{1}}^{t_{2}} \varphi[t(1+\epsilon)] \varphi^{\prime}[t] d t}{\sqrt{\int_{t_{1}}^{t_{2}} \varphi^{2}[t(1+\epsilon)] d t \int_{t_{1}}^{t_{2}} \varphi^{\prime 2}[t] d t}} .
$$

89 This operation is repeated for various values of $\epsilon$. The $\epsilon$ value which maximizes $C C$ is named $\theta$, and corresponds to the apparent relative velocity change. We also define $K d=1-C C(\theta)$, 
which corresponds to the residual decorrelation between the two waveforms, after correction of the phase change. The study of $K d$ constitutes the Coda Wave Decorrelation [Cowan et al., 2002, Larose et al., 2010] (in fluids filled with a suspension of moving scatterers, Diffuse Acoustic Wave Spectroscopy gives $\theta=0$ and $K d \neq 0$ ). It is important to note that $K d$ and $\theta$ are computed for a given time window $\left[t_{1}, t_{2}\right]$ in the coda. The position of this window yields information about the region of the medium being probed. Indeed, the later we look in the signal, the larger and deeper is the area in which waves have traveled. If the changes were homogeneous in the medium, $\theta$ and $K d$ would be the same for any window position. $\theta$, the apparent velocity change, would then become the actual homogeneous relative velocity change taking place in the medium. This is obviously not the case here.

We show in Fig. 3 the evolution of $K d$ and $\theta$ versus the water front elevation, for the observation window $[0.4 \mathrm{~ms}, 0.8 \mathrm{~ms}]$. Error bars in Fig. 3 correspond to the error on $\theta$ induced in the signal processing (Eq. 1) by the presence of decorrelation. We used for its calculation the formula given by [Weaver et al., 2011] which shows that this error, beside increasing with $K d$, also depends on the time-window length and position, along with other parameters such as central frequency and bandwidth.
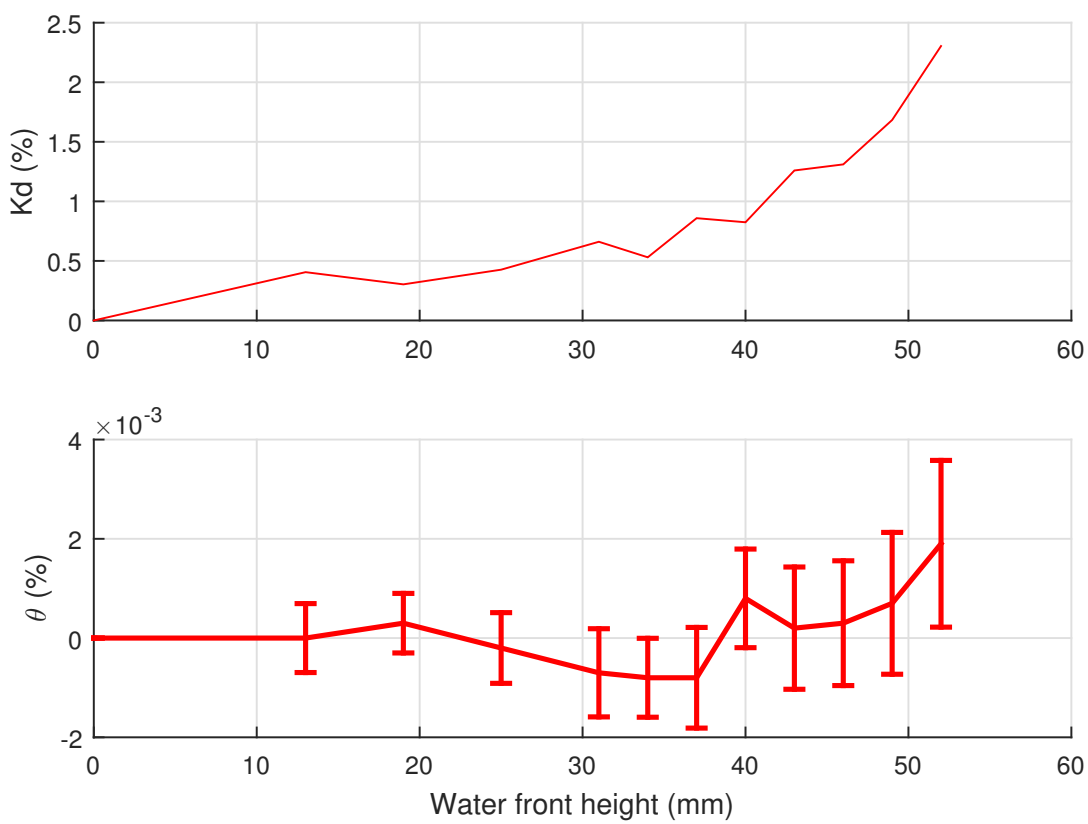

Figure 3: Evolution of stretching observables with water progression in sand.

It is clear in Fig. 3 that $K d$ is increasing with water elevation. It also increases with the observation time in the coda (not shown here), which is coherent since the later we look in the coda, the deeper waves have propagated and the longer they have interacted with changes. This proves that $K d$ is sensitive to water presence. $\theta$ results are however more difficult to 
interpret. Although we can observe variations with water level, they are not much higher than the estimated error bars, which suggests that changes in the waveform are dominating those in phase. In other words, in the case of water imbibition in sand, structural changes occurring at the grain scale are more important than mechanical changes (effective velocity) occurring in the mesoscopic effective medium. CWD hence is a better indicator of water presence in sand than CWI. In the following sections, we confront this observation with other experiments results, as water imbibition in concrete, snow melt infiltration in rocky glaciers, or lava migration in volcanoes.

\section{Monitoring of water imbibition in concrete}

In the litterature, experimental works [Garnier et al., 2013, Abraham et al., 2012, Popovics, 2005, Balayssac et al., 2012] show variations of ballistic ultrasonic waves velocities of $10 \%$ when homogeneous water saturation varies from $35 \%$ to $100 \%$. In the case of a non homogeneous change, for which the medium perturbation would be smaller, the higher sensitivity of coda waves could be an important asset to detect or locate the changes.

We here present the results of an experiment originally designed to image a water content gradient, in a concrete slab submitted to capillary imbibition. Concrete characteristics are reported in Table 1 and a schematic description of the experimental setup is given in Fig. 4.

\begin{tabular}{|c|c|}
\hline Max. aggregate size & $14 \mathrm{~mm}$ \\
\hline Water/cement ratio & 0.8 \\
\hline Density & $2250 \mathrm{~kg} \mathrm{~m}^{-3}$ \\
\hline Open porosity & $18 \%$ \\
\hline
\end{tabular}

Table 1: Concrete characteristics.

Before the experiment, the concrete slab $(600 \times 260 \times 150 \mathrm{~mm})$ is partially dried during several weeks and is then water tight sealed, to ensure a uniform sample without initial water content gradient. The initial (homogeneous) saturation is about $35 \%$, which places us in the saturation regime marked by an increase of ballistic waves velocity, according to [Abraham et al., 2012]. At the beginning of the experiment, the inferior side is submerged in water. Supports have a very limited contact area to limit acoustical transfer in other solids. The fluid level above the bottom of the slab is maintained constant to around $1 \mathrm{~cm}$ so the water pressure underneath the sample is close to the air pressure just above the sample. The resulting pressure difference is hence constant in time, about 1 mbar, which is considered sufficiently small to assume a nonforced imbibition. In order to provoke a vertical phenomenon, lateral sides are covered with 
waterproof resin while the top one is free. To monitor the water gradient's evolution, four moisture sensors (thermo-hygrometric) are placed in the concrete sample at different heights, several weeks before. The experiment is carried out in a climatic chamber maintained at constant temperature. Ambient air temperature along with air humidity level are recorded.

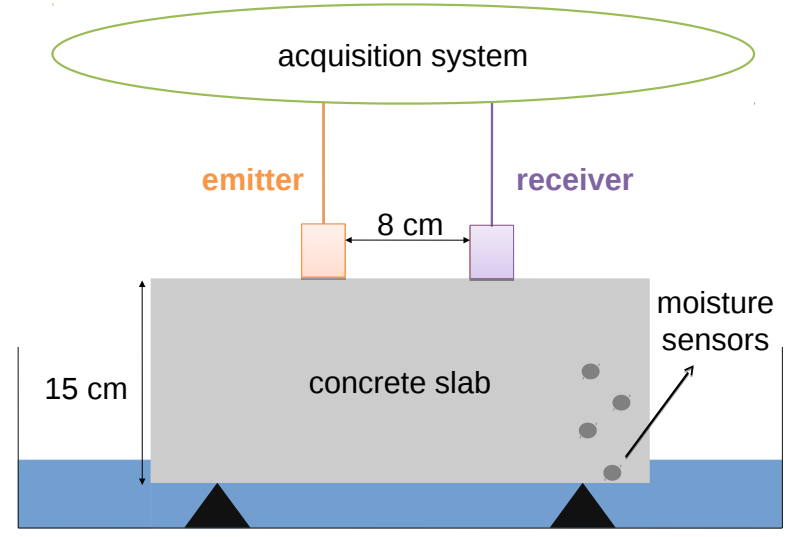

Figure 4: Experimental set-up.

Water imbibition in concrete is monitored using ultrasonic coda.

Ultrasonic emitters and receivers, glued on the top and one lateral side of the slab, retrieve impulse responses in a $200-400 \mathrm{kHz}$ frequency range. These responses are regularly measured in time during the imbibition. For each sensor couple, the first signal measured after the beginning of the imbibition is defined as the reference. We then use the stretching technique, described in the previous section, to compare each of the following signals to this reference.

These comparisons gives us two observables: the apparent relative velocity change $\theta$ and the waveform decorrelation $K d$, whose evolution during imbibition are given in Fig. 5, for one source-receiver couple located on the top side of the block (see Fig. 4).

We first observe in Fig. 5 the difference of magnitude between the two observables. $\theta$ presents indeed very weak variations. This particular point was not expected. According to [Abraham et al., 2012], the (ballistic) relative velocity change in the fully saturated part of the slab should be about $10 \%$. Local variations, much smaller than this, have been detected using CWI [Zhang et al., 2016]. The fact that we are here not able to detect such an important change is due to the high level of waveform decorrelation. Indeed, coda velocity is a property of the effective medium, whereas decorrelation is related to micro-structural changes. Here, these changes are too important to support the hypothesis of a continuously changing effective medium. And comparing velocities during these changes has little meaning. In other words, the conditions of CWI are not respected, particularly the hypothesis of path conservation. For the velocity change to be properly definied in the material, the change should be at a scale much 

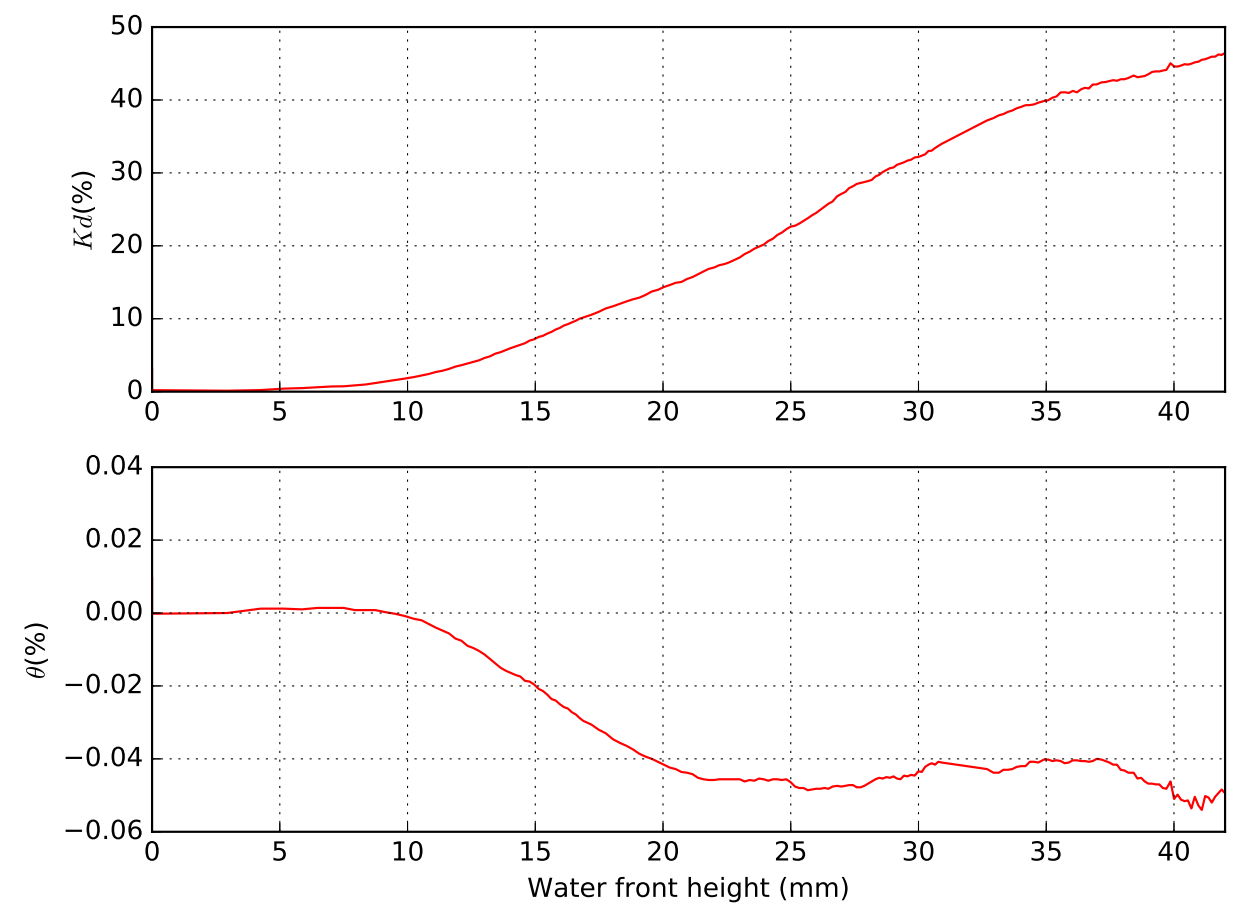

Figure 5: Evolution of stretching observables during water imbibition in concrete.

smaller than the wavelength.

As in previous section, $K d$ is hence found to be much more sensitive to water presence than $\theta$. In the following section, we attempt to use this observation to bring new interpretations in other works, involving fluid in porous media, in a geophysical context.

\section{Insights and perspectives from geophysical works}

\subsection{Rocky glaciers}

As a field aiming to study the modifications of surface wave propagation related to external environmental changes, environmental seismology [Larose et al., 2015] may also be an interesting tool for permafrost destabilization assessment. In this view, CWI and CWD are now used to monitor physical evolutions of rocky glaciers. As the most prominent features in alpine permafrost, and a common occurrence in arctic regions [Schoeneich et al., 2015], rocky glaciers are tongue-shaped bodies of frozen debris matrices with interstitial ice [Arenson et al., 2002]. This porous medium with important heterogeneities is seasonally subject to snow melt infiltration.

We here focus on the field work conducted on the Gugla rocky glacier site (Wallis, Switzerland), where the first seismological monitoring of a rocky glacier takes place. Six seismic sensors record permanent ambient noise data since Autumn 2015. Correlations between recordings of two sensors located along a cross section of the rocky glacier, allow to retrieve the associated Green 
function, which exhibits coda. Indeed, rocky glacier are heterogeneous media, thus causing multiple scattering at high frequencies (here from 10 to $14 \mathrm{~Hz}$ ). Relative velocity changes occuring in the medium can thus be measured, by applying the stretching method between a coda retrieved in a reference period (corresponding to an initial stable state of the medium) to the coda from the day of interest. The two observables (relative velocity change of the Rayleigh wave $d V / V$ and decorrelation $K d$ ) have been linked to meteorological variables [Guillemot et al., ] (article in preparation). Schematic representation of these observables variations are presented in Fig. 6 .

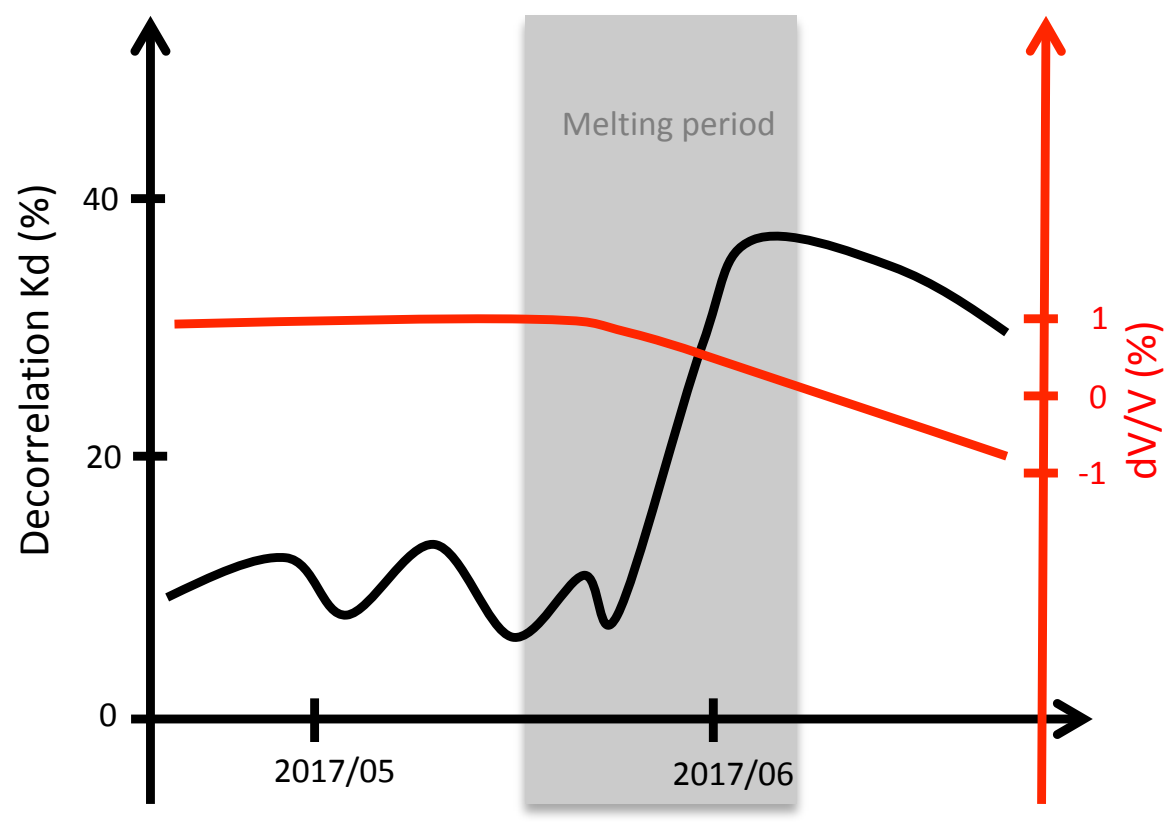

Figure 6: Schematic representation of $K d$ and $\theta$, measured in Gugla-Breithorn rock glacier (Switzerland) during a snow melting period, where melted snow is infiltrating through the porous structure. Curves interpreted from [Guillemot et al., ] (article in preparation).

We can observe a velocity drop during the melting period, and a simultaneous decorrelation event. Whereas $K d$ seems to vary with the volume of water (rain and melted snow) infiltrating in surface porous layers, $d V / V$ variations exhibit better correlation with snow depth evolution [Guillemot et al., ].

This study thus confirms the two findings presented in previous sections. First, decorrelation is sensitive to pore filling, and can be an interesting indicator to monitor water content changes. Secondly, velocity doesn't seem to be very sensitive to fluid infiltration. However in this case, $d V / V$ is related to rigidity or density changes, and thus can be relevant as precursor of a global destabilization of rocky glacier, due to melting effects (but is smaller than $K d$ in absolute value and, thus, less sensitive). 


\subsection{Volcanoes}

In the field of seismology, CWI and CWD have naturally been used to monitor volcanic areas, whose important heterogeneity makes coda-based methods attractive. We here focus on the work made by [Obermann et al., 2013] on the Piton de la Fournaise. This work is based on records made during six months around two eruptions, and proposes 2D-mapping of the velocity change and the cross section density change. To do this imagery, they define a direct problem involving sensitivity kernels, to link the apparent velocity change $\theta$, measured by stretching, to the actual velocity change distribution. The same goes with the cross section density of changes, which is related to $K d$, and quantifies the micro-structural changes occurring at the scatterer scale. These maps are made at different steps of the eruptions.

In this earlier work, velocity changes are supposed to be generated by soil fracturation and inflation due to movements of the pressurized fluid (magma) underneath. The cross section change is interpreted as cracks opening and development, due to the same pressure fluid, and is found to be a more sensitive indicator.

Although this work is well interpreted, ours permits to bring confirmations to certain hypothesis. The first one is that the velocity is weakly impacted by fluid presence but mostly by microfracturation (at sizes much smaller than the seismic wavelength). The second one is that decorrelation is more sensitive, and actually more spatially resolved, than relative velocity changes. Third, decorrelation - and cross section - could be due, not only to the crack opening, but also to fluid injection.

\section{Conclusion}

In this article, two laboratory experiments have been presented, in which water ascends either in a sand or a concrete medium. For each experiment, ultrasonic waves are emitted and recorded from the sample surface. CWI and CWD methods are applied to the measured coda signals, hence giving phase shift and decorrelation values for various water levels.

While we expected both these observables to be sensitive to water elevation, these experiments have shown that water impacts more importantly the decorrelation. The phase shift, corresponding to the apparent relative velocity change, hardly emerges from noise or has very low values. This observation contrasts with results found in litterature for ballistic waves. Our experiments hence show that the velocity change induced by water on ballistic waves is no longer visible when we decrease the wavelength, because of the impact of water on the scatterers (heterogeneities). 
When looking at the scattered waves, changes in the medium microstructure dominate those in the effective medium.

We have then showed that this simple observation could be retreived in geophysical field studies, such as rocky glacier under snow melt infiltration, and pre-eruptive volcanoe. In those studies, this new considerations bring some ligth on the initial interpretations, and allow better comprehension of the problems.

\section{Acknowledgments}

The first author acknowledge $\mathrm{PhD}$ grant funding from IFSTTAR. This work is partially funded by the "ANR ENDE" and "IDEX - Univ. Grenoble Alpes" projects. We also thank J.B. Legland, G. Villain, and O. Durand for their help on the concrete experiment.

\section{References}

[Abraham et al., 2012] Abraham, O., Piwakowski, B., Villain, G., \& Durand, O. (2012). Noncontact, automated surface wave measurements for the mechanical characterisation of concrete. Construction and Building Materials, 37, 904-915.

[Archie, 1942] Archie, G. (1942). The Electrical Resistivity Log as an Aid in Determining Some Reservoir Characteristics. Transactions of the AIME, 146(01), 54-62.

[Arenson et al., 2002] Arenson, L., Hoelzle, M., \& Springman, S. (2002). Borehole deformation measurements and internal structure of some rock glaciers in Switzerland. Permafrost and Periglacial Processes, 13(2), 117-135.

[Balayssac et al., 2012] Balayssac, J. P., Laurens, S., Arliguie, G., Breysse, D., Garnier, V., Dérobert, X., \& Piwakowski, B. (2012). Description of the general outlines of the French project SENSO - Quality assessment and limits of different NDT methods. Construction and Building Materials, 35, 131-138.

[Barker \& Moore, 1998] Barker, R. \& Moore, J. (1998). The application of time-lapse electrical tomography in groundwater studies. The Leading Edge, 17(10), 1454-1458.

[Benson, 1991] Benson, R. C. (1991). Remote Sensing and geophysical methods for evaluation of subsurface conditions. In D. M. Nielsen (Ed.), Practical Handbook of Environmental Site Characterization and Ground-Water Monitoring (pp. 143-194). Lewis Publishers Inc., Chelsea, Michigan. 
[Brenguier et al., 2008] Brenguier, F., Campillo, M., Hadziioannou, C., Shapiro, N. M., Nadeau, R. M., \& Larose, E. (2008). Postseismic relaxation along the San Andreas fault at Parkfield from continuous seismological observations. Science, 321(5895), 1478-1481.

[Cowan et al., 2002] Cowan, M. L., Jones, I. P., Page, J. H., \& Weitz, D. A. (2002). Diffusing acoustic wave spectroscopy. Physical Review E - Statistical, Nonlinear, and Soft Matter Physics, 65(6), 1-23.

[Garnier et al., 2013] Garnier, V., Piwakowski, B., Abraham, O., Villain, G., Payan, C., \& Chaix, J. F. (2013). Acoustic techniques for concrete evaluation: Improvements, comparisons and consistency. Construction and Building Materials, 43, 598-613.

[Grêt et al., 2006] Grêt, A., Snieder, R., \& Scales, J. (2006). Time-lapse monitoring of rock properties with coda wave interferometry. Journal of Geophysical Research: Solid Earth, $111(3), 1-11$.

[Guillemot et al., ] Guillemot, A., Baillet, L., Helmstetter, A., Larose, É., \& Mayoraz, R. Seismic monitoring of the Gugla-Breithorn rock glacier (Switzerland) - In preparation.

[Jia, 2004] Jia, X. (2004). Codalike multiple scattering of elastic waves in dense granular media. Physical Review Letters, 93(15), 8-11.

[Jia et al., 2009] Jia, X. P., Laurent, J., Khidas, Y., \& Langlois, V. (2009). Sound scattering in dense granular media. Chinese Science Bulletin, 54(23), 4327-4336.

[Konstantaki et al., 2013] Konstantaki, L., Carpentier, S., Garofalo, F., Bergamo, P., \& Socco, L. (2013). Determining hydrological and soil mechanical parameters from multichannel surface-wave analysis across the Alpine Fault at Inchbonnie, New Zealand. Near Surface Geophysics, 11(1983), 435-448.

[Larose et al., 2015] Larose, E., Carrière, S., Voisin, C., Bottelin, P., Baillet, L., Guéguen, P., Walter, F., Jongmans, D., Guillier, B., Garambois, S., Gimbert, F., \& Massey, C. (2015). Environmental seismology: What can we learn on earth surface processes with ambient noise? Journal of Applied Geophysics, 116, 62-74.

[Larose \& Hall, 2009] Larose, E. \& Hall, S. (2009). Monitoring stress related velocity variation in concrete with a 2.10-5 relative resolution using diffuse ultrasound. The Journal of the Acoustical Society of America, 125(4), 1853-1856. 
[Larose et al., 2010] Larose, E., Planes, T., Rossetto, V., \& Margerin, L. (2010). Locating a small change in a multiple scattering environment. Applied Physics Letters, 96(20), 204101.

[Lobkis \& Weaver, 2003] Lobkis, O. I. \& Weaver, R. L. (2003). Coda-Wave Interferometry in Finite Solids: Recovery of P-to-S Conversion Rates in an Elastodynamic Billiard. Physical Review Letters, 90(25), 254302.

[Lu \& Sabatier, 2009] Lu, Z. \& Sabatier, J. M. (2009). Effects of Soil Water Potential and Moisture Content on Sound Speed. Soil Science Society of America Journal, 73(5), 1614.

[Obermann et al., 2013] Obermann, A., Planès, T., Larose, E., \& Campillo, M. (2013). Imaging preeruptive and coeruptive structural and mechanical changes of a volcano with ambient seismic noise. Journal of Geophysical Research: Solid Earth, 118(12), 6285-6294.

[Popovics, 2005] Popovics, S. (2005). Effects of uneven moisture distribution on the strength of and wave velocity in concrete. Ultrasonics, 43(6), 429-434.

[Schoeneich et al., 2015] Schoeneich, P., Bodin, X., Echelard, T., Kaufmann, V., KellererPirklbauer, A., Krysiecki, J. M., \& Lieb, G. K. (2015). Velocity Changes of Rock Glaciers and Induced Hazards. In Engineering Geology for Society and Territory - Volume 1 (pp. 223-227). Cham: Springer International Publishing.

[Sens-Schönfelder \& Wegler, 2006] Sens-Schönfelder, C. \& Wegler, U. (2006). Passive image interferemetry and seasonal variations of seismic velocities at Merapi Volcano, Indonesia. Geophysical Research Letters, 33(21), 1-5.

[Snieder, 2006] Snieder, R. (2006). The theory of coda wave interferometry. Pure and Applied Geophysics, 163(2-3), 455-473.

[Snieder et al., 2002] Snieder, R., Grêt, A., Douma, H., \& Scales, J. (2002). Coda wave interferometry for estimating nonlinear behavior in seismic velocity. Science, 295(5563), 2253-2255.

[Villain et al., 2012] Villain, G., Sbartaï, Z. M., Dérobert, X., Garnier, V., \& Balayssac, J.P. (2012). Durability diagnosis of a concrete structure in a tidal zone by combining NDT methods: Laboratory tests and case study. Construction and Building Materials, 37, 893903.

[Weaver et al., 2011] Weaver, R. L., Hadziioannou, C., Larose, E., \& Campillo, M. (2011). On the precision of noise correlation interferometry. Geophysical Journal International, 185(3), $1384-1392$. 
314 [Winkler \& Nur, 1979] Winkler, K. \& Nur, A. (1979). Pore fluids and seismic attenuation in rocks. Geophysical Research Letters, 6(1), 1-4.

[Zhang et al., 2012] Zhang, Y., Abraham, O., Grondin, F., Loukili, A., Tournat, V., Duff, A. L., Lascoup, B., \& Durand, O. (2012). Study of stress-induced velocity variation in concrete under direct tensile force and monitoring of the damage level by using thermally-compensated Coda Wave Interferometry. Ultrasonics, 52(8), 1038-1045.

[Zhang et al., 2018] Zhang, Y., Larose, E., Moreau, L., \& D'Ozouville, G. (2018). Threedimensional in-situ imaging of cracks in concrete using diffuse ultrasound. Structural Health Monitoring, 17(2), 279-284.

[Zhang et al., 2016] Zhang, Y., Planès, T., Larose, E., Obermann, A., Rospars, C., \& Moreau, G. (2016). Diffuse ultrasound monitoring of stress and damage development on a 15-ton concrete beam. The Journal of the Acoustical Society of America, 139(4), 1691-1701. 